\title{
Comment on "Reduced efficacy of marine cloud brightening geoengineering due to in-plume aerosol coagulation: parameterization and global implications" by Stuart et al. (2013)
}

\author{
S. Anand ${ }^{1}$ and Y. S. Mayya ${ }^{2}$ \\ ${ }^{1}$ Health Physics Division, Bhabha Atomic Research Centre, Trombay, Mumbai - 400 085, India \\ ${ }^{2}$ Department of Chemical Engineering, Indian Institute of Technology Bombay (IIT-B), Powai, Mumbai - 400 076, India
}

Correspondence to: Y. S. Mayya (ysmayya@iitb.ac.in)

Received: 22 July 2014 - Published in Atmos. Chem. Phys. Discuss.: 15 September 2014

Revised: 11 December 2014 - Accepted: 11 December 2014 - Published: 21 January 2015

\begin{abstract}
We examine the parameterized model of Stuart et al. (2013) vis-à-vis a diffusion-based model proposed by us earlier (Anand and Mayya, 2011) to estimate the fraction of aerosol particles surviving coagulation in a dispersing plume. While the Stuart et al. approach is based on the solutions to the coagulation problem in an expanding plume model, the diffusion-based approach solves the diffusion-coagulation equation for a steady-state standing plume to arrive at the survival fraction correlations. We discuss the differences in the functional forms of the survival fraction expressions obtained in the two approaches and compare the results for the case studies presented in Stuart et al. (2013) involving different particle emission rates and atmospheric stability categories. There appears to be a better agreement between the two models at higher survival fractions as compared to lower survival fractions; on the whole, the two models agree with each other within a difference of $10 \%$. The diffusion-based expression involves a single exponent fit to a theoretically generated similarity variable combining the parameters of the problem with inbuilt exponents and hence avoids the multi-exponent parameterization exercise. It also possesses a wider range of applicability in respect of the source and atmospheric parameters as compared to that based on parameterization. However, in the diffusion model, the choice of a representative value for the coagulation coefficient is more prescriptive than rigorous, which has been addressed in a more satisfactory manner by the parameterization method. The present comparative exercise, although limited in scope, confirms the importance of aerosol microphysical processes envisaged by Stuart et al. for cloud brightening applications.
\end{abstract}

In a larger context, it seems to suggest that either of the two forms of expressions might be suitable for incorporation into global-/regional-scale air pollution models for predicting the contribution of localized sources to the particle number loading in the atmosphere.

\section{Introduction}

A parameterization scheme is provided by Stuart et al. (2013) (hereafter, S13) to assess the loss of particle number concentration by coagulation in plumes for cloud-resolving and global models. The authors numerically solve the coagulation problem in a dispersing plume and employ a multi-exponent parameterization scheme to obtain a semiempirical equation by fitting their multi-shelled Gaussian plume model to five atmospheric dispersion- and sourcerelated parameters. The fitted formula is then used to estimate the fraction of particles surviving coagulation (survival fraction) within a dispersing plume volume. The choice of the functional form of empirical equation in S13 is based on the survival fraction formula provided earlier by Turco and $\mathrm{Yu}(1997)$ within the framework of solving the coagulation equation in a volume which is expanding at a prescribed rate in time. The simplifying feature of the Turco and $\mathrm{Yu}$ model (1997) is that it replaces the gradient-driven nature of the dispersion process by a purely time-dependent term leading to an analytically tractable solution to the survival fraction. 
As an alternative to the above approach, Anand and Mayya $(2009,2011)$ have developed a formalism based on solving the coagulation-diffusion equation for estimating the survival fraction of aerosols in dispersing puffs and plumes. In their 2011 work, they specifically addressed the issue of particle number survival fraction in a standing plume, maintained by a steady emission source, by combining turbulent diffusion and advection with coagulation through an equation of the form

$v_{\mathrm{w}} \frac{\partial N}{\partial x}=\frac{v_{\mathrm{w}}}{4} \frac{\mathrm{d} \sigma^{2}}{\mathrm{~d} x}\left[\frac{1}{r} \frac{\partial}{\partial r}\left(r \frac{\partial N}{\partial r}\right)\right]-\frac{K_{\mathrm{c}}}{2} N^{2}$.

In Eq. (1), $N$ is the particle number concentration, $v_{\mathrm{w}}$ is the wind speed, $\sigma$ is the plume width (expressed through a spatially varying turbulent diffusion coefficient), $x$ and $r$ are the downwind and the cross-wind coordinates respectively, and $K_{\mathrm{c}}$ is an effective coagulation coefficient, taken as a sizeindependent constant. The source emission rate provides the flux matching condition at $x=0$. Basically, this model provides a mechanistic basis for dispersion to estimate the survival fraction in an Eulerian framework; further, it directly solves Eq. (1) to obtain the number concentration profile in a standing plume.

Given the steady-state nature of the above approach, the survival fraction is evaluated rather differently as compared to time-dependent expansion problems. It is defined as the ratio of the flux of particles integrated over the entire cross section at a downstream distance $x$ to that emitted in the source domain, in the limit, $x \rightarrow \infty$. A scaling analysis of Eq. (1) showed that the survival fraction $(F)$ is a unique function of a similarity variable $\mu$ (see below) that combines all the parameters of the problem with inbuilt exponents. Further, a limiting analysis indicated that $F$ should possess a functional form of the type $(1+\mu / v)^{-v}$, where $v$ is an exponent to be determined. Upon combining these results with a numerical solution of Eq. (1) for evaluating $v$ through a single exponent fit, the survival fraction was then represented in terms of the variable $\mu$, in the following form:

$F=\frac{1}{(1+1.32 \mu)^{0.76}}$,

where

$\mu=\frac{K_{\mathrm{c}} P}{6 \sqrt{3} v_{\mathrm{w}}\left(2 R_{\mathrm{S}}\right)^{4 / 3}(C \varepsilon)^{1 / 3}}$,

where $K_{\mathrm{c}}$ is the effective coagulation coefficient, $P$ is the number emission rate, $v_{\mathrm{w}}$ is the wind velocity, $R_{\mathrm{S}}$ is the emission stack radius (plume radius at the source of emission), $C$ is a constant (0.8), and $\varepsilon$ is the turbulent kinetic energy dissipation rate. As in the case of S13, the present result also involves five parameters all combined in a single variable $\mu$. However, there are subtle differences: the present model involves two parameters $\left(v_{\mathrm{w}}\right.$ and $\left.\varepsilon\right)$ to describe atmospheric conditions, whereas $\mathrm{S} 13$ account for this through $v_{\mathrm{w}}$ and stability category. On the other hand, the present model captures coagulation characteristics through a single parameter $K_{\mathrm{c}}$, whereas $\mathrm{S} 13$ use polydispersity index $(\sigma)$ and particle diameter $\left(D_{\mathrm{p}}\right)$ separately to account for coagulation. Since a significant part of the coagulation effect is expected to occur near the source region, where the particle concentration will be the highest, we use the value of the effective coagulation coefficient $\left(K_{\mathrm{c}}\right)$ of the initial aerosol spectrum. This may be viewed as a model prescription which may not be entirely satisfactory for an evolving aerosol spectrum. An important point about the present model is that the survival fraction formula (Eq. 2) can be applied beyond the fitting range of input values tested in S13, and it provides a general framework to coagulation of aerosols in plumes (e.g. forest fires, volcanic emissions).

The quantity $\varepsilon$, the turbulent kinetic energy dissipation rate (Table 1), may be estimated for different atmospheric stability classes through the well-known relationships of atmospheric boundary layer theory (Han et al., 2000). In Table 1, $x=\left[1-15 \frac{z}{L}\right]^{1 / 4}, L$ is Monin-Obukhov length, $u_{*}$ is the friction velocity (Stull, 1988), $z$ is the height of release, $z_{0}$ is the roughness length, $k$ is the van Karman constant (0.4), and $u$ is the wind velocity. The Monin-Obukhov length $(L)$ is obtained using a fitting expression (Seinfeld and Pandis, 2006) for various stability categories and roughness length. The $L$ values obtained corresponding to a $z_{0}$ of $0.02 \mathrm{~m}$ (oceanic surface) are -11.6 for unstable, $\infty$ for neutral, and 10.4 for stable categories, and these are used in the present study.

\section{Results and discussion}

We now compare the estimates of the survival fractions from these two models using the case studies described in $\mathrm{S} 13$ and the values presented in their Table 1 for the wind speed, particle emission rates and stack radius. In the present calculations, the atmospheric stability classes A, B, and C have been combined into a single (unstable) category, and the classes $\mathrm{E}$ and F have been combined into one "stable" category. The category D (neutral) has been retained as such.

The results of the survival fractions obtained with the two approaches are in Table 2. The survival fraction values obtained for "Minimum", "Base", and "Maximum" cases (Table 2) correspond respectively to the minimum, base, and maximum of all the five parameters mentioned in Table 1 of S13. The number of survival fractions estimated from the present model varies from 0.36 to 0.62 , thereby confirming the important role of aerosol microphysical processes as envisaged by $\mathrm{S} 13$, in significantly altering the source to receptor transfer of particles for cloud brightening applications.

Except in the E/F category for the "maximum" case, the survival fraction estimates from the two approaches for all other cases are rather close to each other. Both the models seem to predict similar trends: survival fractions are lower 
Table 1. Friction velocity $\left(u_{*}\right)$ and turbulent kinetic energy (TKE) dissipation rate $(\varepsilon)$ for various atmospheric stability categories. (See text for definition of quantities $x, L$, and $z_{0}$.)

\begin{tabular}{lll}
\hline Stability category & Friction velocity $\left(u_{*}\right), \mathrm{m} \mathrm{s}^{-1}$ & TKE dissipation rate $(\varepsilon), \mathrm{m}^{2} \mathrm{~s}^{-3}$ \\
\hline Unstable & $k u\left[\ln \left(\frac{z}{z_{0}}\right)-2 \ln \left(\frac{1+x}{2}\right)-\ln \left(\frac{1+x^{2}}{2}\right)+2 \tan ^{-1} x-\frac{\pi}{2}\right]^{-1}$ & $\frac{u_{*}^{3}}{k z}\left(1+0.5\left|\frac{z}{L}\right|^{2 / 3}\right)^{3 / 2}$ \\
Neutral & $k u\left[\ln \left(\frac{z}{z_{0}}\right)\right]^{-1}$ & $\frac{u_{*}^{3}}{k z}\left(1.24+4.3 \frac{z}{L}\right)\left(1-0.85 \frac{z}{h}\right)^{3 / 2}$ \\
Stable & $k u\left[\ln \left(\frac{z}{z_{0}}\right)+\frac{4.7\left(z-z_{0}\right)}{L}\right]^{-1}$ & same as above \\
\hline
\end{tabular}

Table 2. Number survival fraction in a plume obtained using the two models.

\begin{tabular}{lllllll}
\hline \multirow{2}{*}{$\begin{array}{c}\text { Stability } \\
\text { category }\end{array}$} & \multicolumn{5}{c}{ Number survival fraction } \\
\cline { 2 - 7 } & \multicolumn{5}{c}{ Minimum } & \multicolumn{2}{c}{ Base } & \multicolumn{2}{c}{ Maximum } \\
\cline { 2 - 7 } & Eq. (5) of S13 & Eq. (2) & Eq. (5) of S13 & Eq. (2)* & Eq. (5) of S13 & Eq. (2)* \\
\hline $\mathrm{A}$ & 0.629 & \multirow{2}{*}{0.562} & 0.515 & \\
$\mathrm{~B}$ & 0.626 & 0.621 & 0.549 & 0.544 & 0.497 & 0.495 \\
$\mathrm{C}$ & 0.589 & & 0.492 & & 0.429 & \\
\hline $\mathrm{D}$ & 0.547 & 0.507 & 0.436 & 0.43 & 0.368 & 0.384 \\
\hline $\mathrm{E}$ & 0.505 & \multirow{2}{*}{0.481} & 0.379 & 0.405 & 0.303 & \multirow{2}{*}{0.361} \\
$\mathrm{~F}$ & 0.404 & & 0.266 & & 0.191 & \\
\hline
\end{tabular}

* Eq. (47) of Anand and Mayya (2011) is reproduced as Eq. (2) in the present work.

for increasing emission rate and/or increasing atmospheric stability. There appears to be better agreement between the two models at higher survival fractions and relatively poorer agreement at lower survival fractions. On the whole, it is still remarkable that both the models are close to each other within $10 \%$.

However, it must be reiterated that the two models are based on different formulational premises and predict different forms of the survival fraction on source-related and turbulence-related parameters. For example, in the limit of low particle emission rate $(P \rightarrow 0)$, Eq. (2) of our model predicts that the depleted/consumed particle fraction $(1-F) \rightarrow \mu \propto P$, whereas Eq. (5) of S13 predicts a power-law dependence of the form $(1-F) \propto P^{c}$, with $c$ ranging from 0.51 to 0.76 . On the other hand, in the limit of large emission rates, both the models predict a power-law decline of $F$ with respect to $P$, with similar, if not identical, powers. It will be rewarding to explore the implications of these approaches in the general context of atmospheric aerosols for estimating the contribution of various anthropogenic sources to background particles. Seen from this perspective, the diffusion-based model has the inherent capability to generate a similarity variable with inbuilt exponents for the parameters and hence avoids the multi-exponent parameterization exercise. However, the limitation of the diffusion model is that it does not provide a rigorous framework for the choice of a representative value for the coagulation coefficient in an evolving aerosol system, which has been addressed in a more satisfactory manner by the parameterization method (S13). Notwithstanding these issues, the present numerical comparisons, although limited in scope, seem to suggest that either of the two forms of expressions might be suitable for incorporation into global-/regional-scale air pollution models for predicting the contribution of localized sources to the particle number loading in the atmosphere.

Edited by: V.-M. Kerminen

\section{References}

Anand, S. and Mayya, Y. S.: Coagulation in a diffusing Gaussian aerosol puff: Comparison of analytical approximations with numerical solutions, J. Aerosol Sci., 40, 348-361, 2009.

Anand, S. and Mayya, Y. S.: A simplified approach for solving coagulation-diffusion equation to estimate atmospheric background particle number loading factors contributed by emissions from localized sources, Atmos. Environ., 45, 4488-4496, 2011.

Han, J., Arya, S. P., Shen, S., and Lin, Y.-L.: An Estimation of Turbulent Kinetic Energy and Energy Dissipation Rate Based on Atmospheric Boundary Layer Similarity Theory, NASA/CR-2000210298, 3-5, 2000.

Seinfeld, J. H. and Pandis, S. N.: Atmospheric Chemistry and Physics: From Air Pollution to Climate Change, 2nd Edition, Wiley \& Sons, Inc., New York, 751 pp., 2006. 
Stuart, G. S., Stevens, R. G., Partanen, A.-I., Jenkins, A. K. L., Korhonen, H., Forster, P. M., Spracklen, D. V., and Pierce, J. R.: Reduced efficacy of marine cloud brightening geoengineering due to in-plume aerosol coagulation: parameterization and global implications, Atmos. Chem. Phys., 13, 10385-10396, doi:10.5194/acp-13-10385-2013, 2013.
Stull, R. B.: An Introduction to Boundary Layer Meteorology, Kluwer Academic Publishers, Dordrecht, the Netherlands, Chapter 9, 1988.

Turco, R. and Yu, F.: Aerosol invariance in expanding coagulating plumes, Geophys. Res. Lett., 24, 1223-1226, 1997. 